\title{
Editorial
}

\section{New Developments in Time-Delay Systems and Its Applications in Engineering}

\author{
Guangdeng Zong, ${ }^{1}$ Wei Xing Zheng, ${ }^{2}$ Ligang $\mathrm{Wu},{ }^{3}$ and Yang $\mathrm{Yi}^{4}$ \\ ${ }^{1}$ Research Institute of Automation, Qufu Normal University, Qufu 273165, China \\ ${ }^{2}$ School of Computing, Engineering and Mathematics, University of Western Sydney, Penrith, NSW 2751, Australia \\ ${ }^{3}$ Space Control and Inertial Technology Research Center, Harbin Institute of Technology, Harbin 150001, China \\ ${ }^{4}$ College of Information Engineering, Yangzhou University, Yangzhou 225009, China
}

Correspondence should be addressed to Guangdeng Zong; zonggdeng@yahoo.com.cn

Received 27 June 2013; Accepted 27 June 2013

Copyright (C) 2013 Guangdeng Zong et al. This is an open access article distributed under the Creative Commons Attribution License, which permits unrestricted use, distribution, and reproduction in any medium, provided the original work is properly cited.

A system is said to have a delay when the rate of variation in the system state depends on past states. Such a system is called a time-delay system. Over the past several decades, there has been extensive concern in time-delay systems in the field of control theory and engineering. On one hand, time delays are inherent in various engineering systems such as long transmission lines in pneumatic systems, networked control systems, nuclear reactors, rolling mills, hydraulic systems, and manufacturing. On the other hand, it has been recognized that time delay is often a source of generation of oscillation, instability, or poor control performance of underlying control systems. Therefore, for time-delay systems, there are many challenging issues, for example, stability analysis, stabilization, $H_{\infty}$ control, robust performance analysis, model identification problem, and antidisturbance control, that need to be solved.

This special issue contains twenty-one papers, of which seven are related to stability analysis and stabilization of different time-delay systems and four concern networked control systems with time-delay. Six papers discuss antidisturbance problem and $H$-infinite robust control for various time-delay systems. There is also a single paper focusing on collaboration control for multiagent systems with sampling delay. Another paper deals with online identification of multivariable discrete time-delay systems. Finally, two papers cover the switched models with state delays.

"Stability and $L_{1}$ gain analysis for positive $2 D$ systems with state delays in the Roesser model" by Z. Duan et al. concerns the state-delay term described by the Roesser model and shows the sufficient conditions of delay-dependent stability for positive 2D systems. "New exponential stability conditions of switched BAM neural networks with delays" by Y. Yang constructs a new switching dependent Lyapunov-Krasovskii function and solves the exponential stability problem for a class of discrete-time switched BAM neural networks with time delay, while "Stability analysis for delayed neural networks: reciprocally convex approach" by $\mathrm{H}$. Yu et al. investigates the global stability problem for a class of continuous neural networks with time-varying delay by using reciprocally convex combination approaches. "Stability analysis of a harvested prey-predator model with stage structure and maturation delay" by C. Liu et al. proposes a harvested prey-predator model to investigate the effects of density dependent maturation delay and discusses the global stability of positive equilibrium by utilizing an iterative technique. " $P$ moment stability of stochastic differential delay systems with impulsive jump and Markovian switching" by L. Gao gives a novel P-moment stability criteria for stochastic differential delay systems. "A simplified descriptor system approach to delay-dependent stability and robust performance analysis for discrete-time systems with time delays" by $\mathrm{F}$. $\mathrm{Xu}$ and D. Li reduces the conservatism of the existing results of time-delay discrete-time systems by removing the redundant matrix variables. "Directly solving special second order delay differential equations using Runge-Kutta-Nyström method" by M. Mechee et al. studies a novel stability analysis method for 
the second order delay differential equations based on the designed Runge-Kutta-Nyström algorithm.

"Robust fault tolerant control for a class of time-delay systems with multiple disturbances" by S. Cao and J. Qiao addresses a robust fault tolerant control approach for a class of nonlinear systems with time-delay, actuator faults, and multiple disturbances using a composite strategy consisting of disturbance observer-based control and fault accommodation. "Enhanced disturbance-observer-based control for a class of time-delay system with uncertain sinusoidal disturbances" by $\mathrm{X}$. Wen provides a thorough study on disturbance-observerbased control (DOBC) for a class of time-delay systems under uncertain sinusoidal disturbances. "Composite disturbance observer-based control and $H_{\infty}$ output tracking control for discrete-time switched systems with time-varying delay" by $\mathrm{H}$. Sun and L. Hou concerns the problem of $H_{\infty}$ output tracking control for discrete-time switched systems with time-varying delay and external disturbances. Furthermore, "Robust $H_{\infty}$ control of uncertain T-S fuzzy time-delay system: a delay decomposition approach" by C. Gong and C. Han concerns with the problem of robust $H_{\infty}$ control of a class of uncertain time-delay fuzzy systems by utilizing the instrumental idea of delay decomposition. "A novel approach to $H_{\infty}$ control design for linear neutral time-delay systems" by $\mathrm{H}$. Xia et al. explores some delay-dependent sufficient conditions of linear time-varying neutral systems by designing the state feedback controller with $H_{\infty}$ performance level. " $H_{\infty}$ control for flexible spacecraft with time-varying input delay" by $\mathrm{R}$. Zhang et al. provides an effective $H_{\infty}$ control algorithm for flexible spacecraft with time-varying control input delay and obtains a more flexible result.

"Performance of networked control systems" by Y. Zhang et al. designs an optimal controller for the new NCSs with data packets dropout and minimizes the infinite performance index at each sampling time. "Control for networked control systems with time delays and packet dropouts" by Y. Wang et al. explores the mean square exponential $H_{\infty}$ performance for NCSs with random delay and packet dropout. "Improving the performance metric of wireless sensor networks with clustering Markov chain model and multilevel fusion" by S. Havedanloo and H. R. Karimi proposes a performance metric evaluation of a distributed detection wireless sensor network with respect to IEEE 802.15.4 standard and improves the performance of network in terms of reliability, packet failure, average delay, and power consumption. "Robust filtering for networked stochastic systems subject to sensor nonlinearity" by $\mathrm{G}$. Wu et al. considers the effects of the sensor saturation, output quantization, and network-induced delay in the network environment and models the random delays as a linear function of the stochastic variable described by a Bernoulli random binary distribution.

"Collaboration control of fractional-order multiagent systems with sampling delay" by H.-Y. et al. Yang investigates the novel collaboration control problems of continuous-time networked fractional-order multiagent systems via sampled control and sampling delay and many sufficient conditions for reaching consensus with sampled data, and sampling delay can be obtained. "Online identification of multivariable discrete time delay systems using a cursive least square algorithm" by S. Bedoui identifies the time delays and the parameters of linear discrete-time delay multivariable systems by using the least square approach. Moreover, "Robust reliable control of uncertain discrete impulsive switched systems with state delays" by X. Li et al. presents the problem of robust reliable control for a class of uncertain discrete impulsive switched systems with state delays and actuator failures. "Sliding mode control based on observer for a class of state-delayed switched systems with uncertain perturbation" by Z. He et al. proposes a state observer-based sliding mode control design methodology for a class of continuous-time state-delayed switched systems with unmeasurable states and nonlinear uncertainties.

It is noted that both the stability analyze method and the $H_{\infty}$ control for complex time-delay systems have always been hot issues in the field of control theory for the last 20 years. Recently, networked control systems (NCSs) have been extensively investigated due to its broad applications in engineering, in which the phenomenon of networkedinduced communication time-delay has received more and more research interests. It is well known that external disturbances originating from various sources exist in almost all controlled systems accompanied by increasingly largescale and complicated industrial processes. Thus, the research of antidisturbance control and disturbance attenuation performance for complex time-delay systems is a challenging problem. On the other hand, the performance analyze for switched systems with time-delay has also have received considerable attention because of their applicability and significance in various areas. In summary, almost all papers in this special issue concern those recent focus and some new developments emerged in the time-delay systems. Moreover, many practical applications can also be found in this special issue, such as truck trailer systems, flexible spacecraft, A4D aircraft, and NCSs.

Of course, the selected topics and papers are not a comprehensive representation of the area of this special issue. Nonetheless, they represent the rich and many faceted knowledge that we have the pleasure of sharing with the readers.

\section{Acknowledgments}

We would like to express appreciation to the authors for their excellent contributions and patience in assisting us. Finally, the fundamental work of all reviewers on these papers is also very greatly acknowledged.

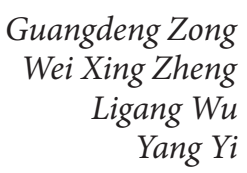




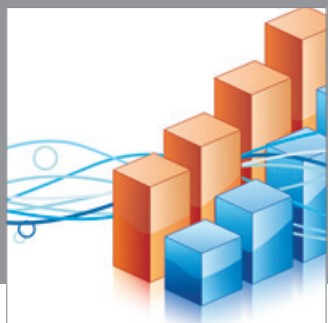

Advances in

Operations Research

mansans

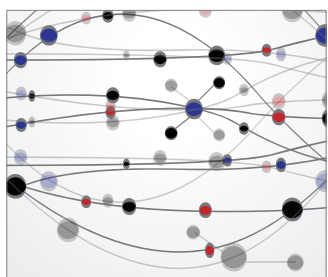

The Scientific World Journal
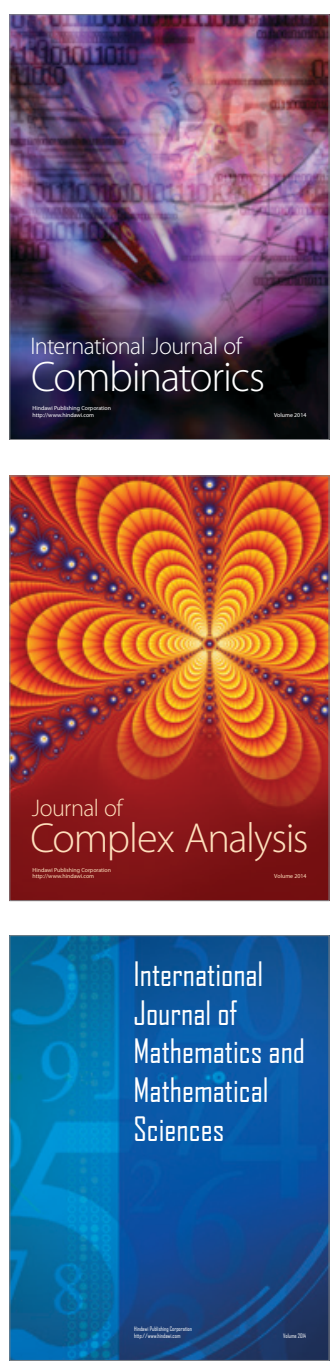
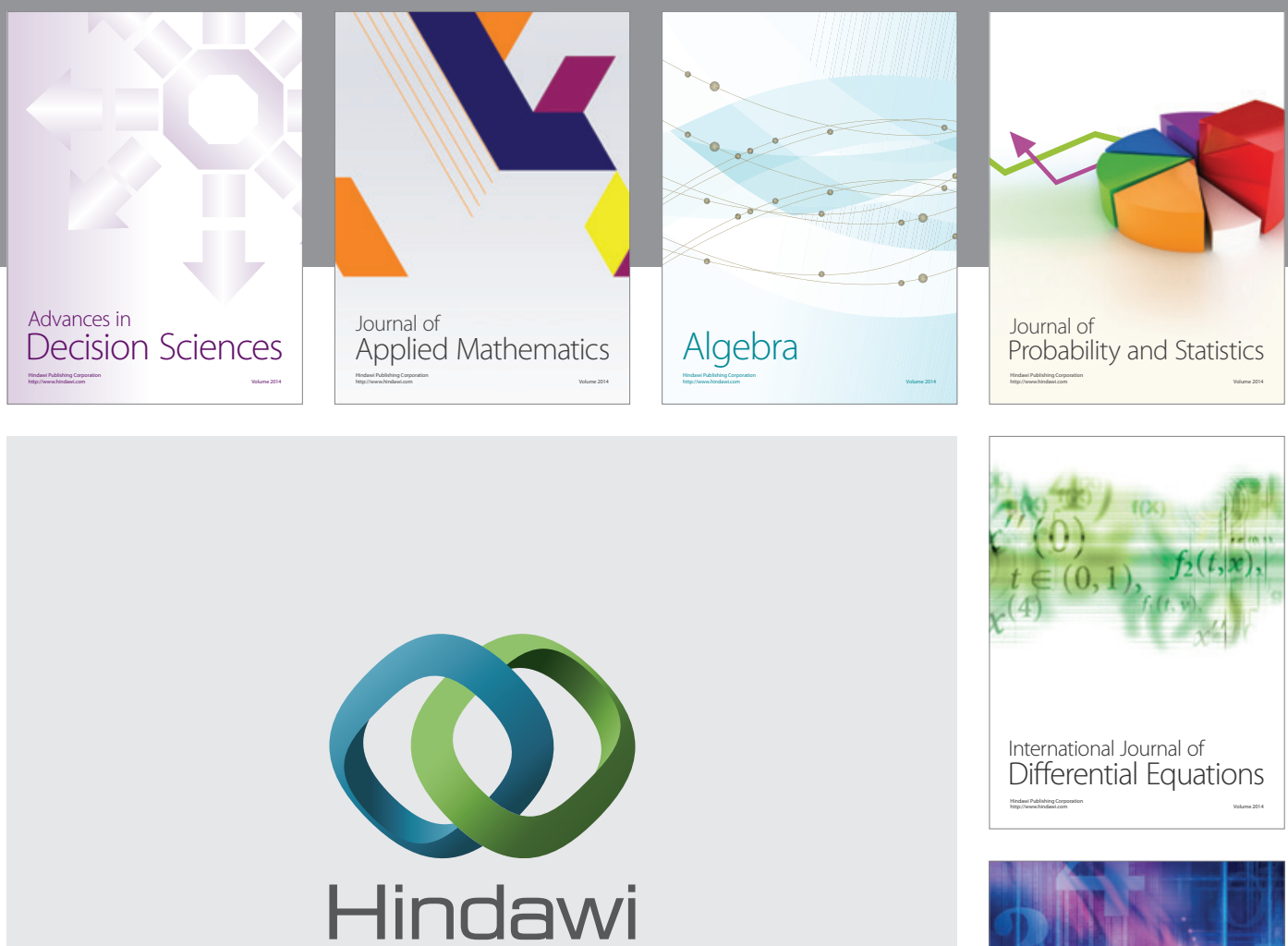

Submit your manuscripts at http://www.hindawi.com
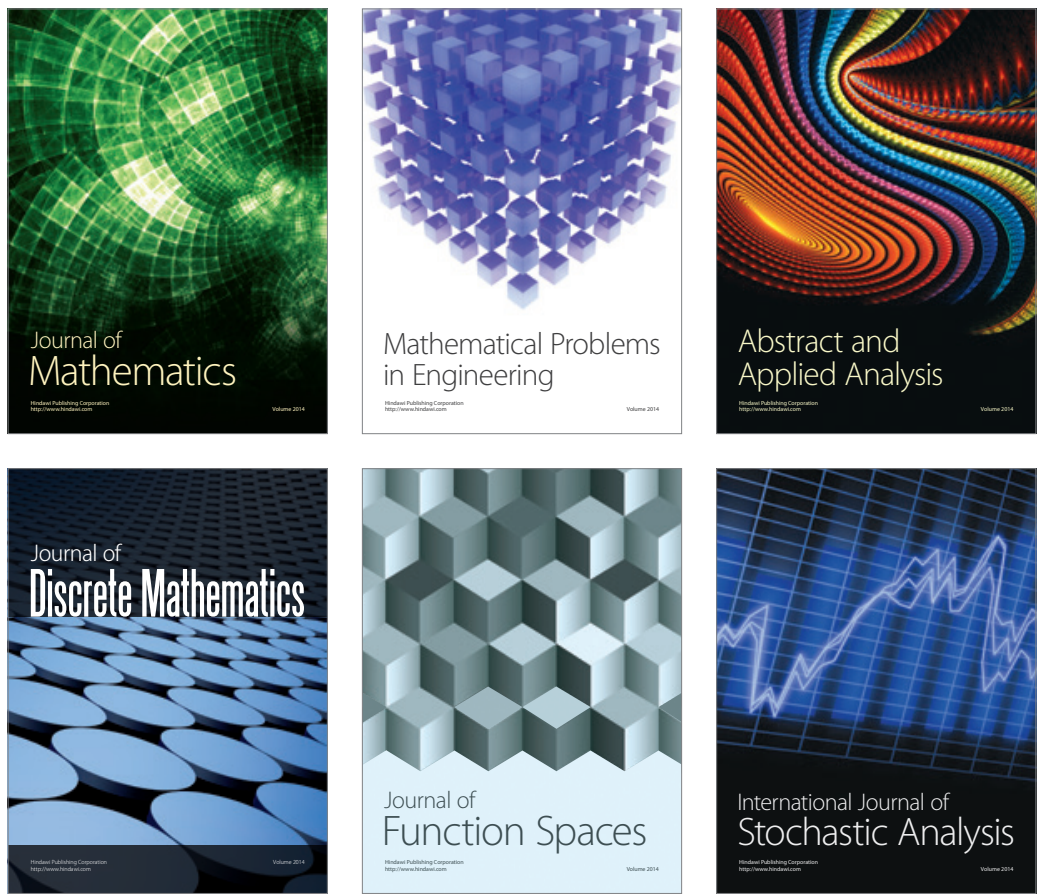

Journal of

Function Spaces

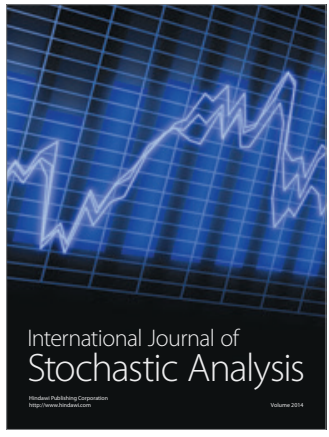

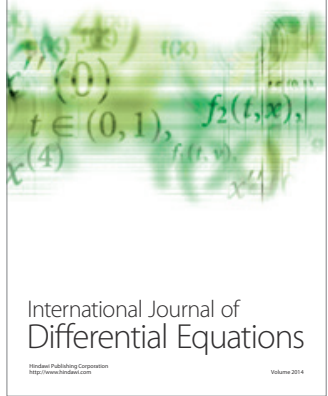
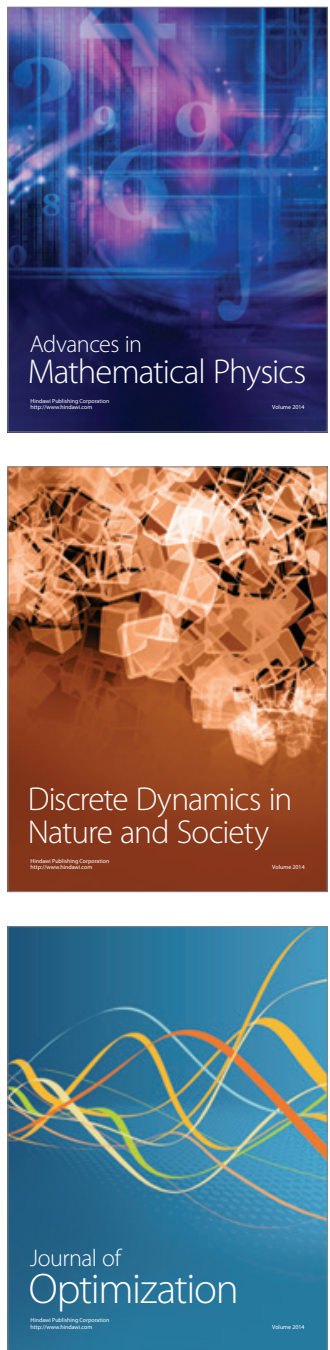